\title{
Avances de la Tecnología Organ-on-a-chip de Riñón hacia Dispositivos de Remplazo de la Función Renal
}

\section{Kidney Organ-on-a-chip Technology Advances towards Renal Function Replacement Devices}

\author{
María Belém García-San Luis ${ }^{1}$, Christian Chapa-González² \\ 1 Instituto Politécnico Nacional \\ 2 Universidad Autónoma de Ciudad Juárez
}

\section{RESUMEN}

La enfermedad renal crónica (ERC) requiere de terapia de reemplazo renal en su última fase, la cual incluye diálisis en cualquiera de sus modalidades y trasplante de riñón. Hasta ahora, la mejor opción es el trasplante renal, sin embargo, la insuficiencia de donadores es la principal limitante, por lo que en los últimos años ha surgido la idea de suplantar la función renal con dispositivos biomédicos. El organ-on-a-chip es una de las principales tecnologías emergentes y se refiere a un sistema biomimético de órganos fisiológicos construido en un chip principalmente de microfluidos. El objetivo de esta revisión es describir los avances de los dispositivos biomédicos de remplazo de la función renal por componentes del riñón, resaltando al final los dispositivos en fases clínicas. La búsqueda de artículos giró en torno a dispositivos microfluídicos (organ-on-a-chip de riñón), que incluyen cultivos de células renales, modelos impresos 3D o bien la combinación de diferentes técnicas. La diversidad de enfoques respecto al abordaje de estudios de la función renal en un organ-on-a-chip conduce a una heterogeneidad en los resultados, sin embargo, luego de clasificar los avances según el componente renal, se proponen diferentes desafíos que deberán resolverse para avanzar en el desarrollo de dispositivos que suplan la función renal..

PALABRAS CLAVE: riñón; dispositivo; organ-on-a-chip; implantable.

\section{ABSTRACT}

Chronic kidney disease (CKD) requires renal replacement therapy in its last phase, which includes dialysis in any of its modalities and kidney transplantation. Until now, the best option is kidney transplantation, however, donor failure is the main limitation. Therefore, in recent years the idea of supplanting kidney function with biomedical devices has emerged. The organ-on-a-chip is one of the main emerging technologies and refers to a biomimetic system of physiological organs built on a chip of mainly microfluidics. The objective of this review is to describe the advances in biomedical devices for the replacement of renal function by kidney components. Also, the devices in clinical phases are highlighted. The search for articles revolved around microfluidic devices (kidney organ-on-a-chip), that include kidney cell cultures, 3D printed models or the combination of different techniques. The diversity of approaches regarding the approach to kidney function studies in an organ-on-a-chip leads to heterogeneity in the results. However, after classifying the advances according to the renal component, we propose different challenges that must be resolved in order to advance in the development of devices that supplant renal function.

KEYWORDS: kidney; device; organ-on-a-chip; implantable.

Correspondencia:

DESTINATARIO: Christian Chapa González

INSTITUCIÓN: Universidad Autónoma de Ciudad Juárez

DIRECCIÓN: Av. del Charro 460 norte, col. Partido Romero, Ciudad Juárez, Chih. C. P. 32310

CORREO ELECTRÓNICO: christian.chapa@uacj.mx
Fecha de recepción: 1 de septiembre de 2020. Fecha de aceptación: 17 de febrero de 2021. Fecha de publicación: 22 de febrero de 2021.
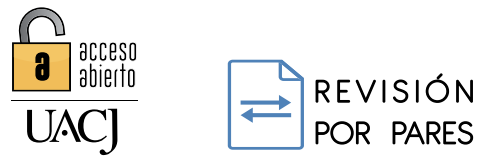

Licencia Creative Commons POR PARES

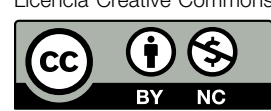




\section{INTRODUCCIÓN}

Los riñones están ubicados en la pared posterior del abdomen, fuera de la cavidad peritoneal, y son los encargados de excretar productos metabólicos de desecho y sustancias químicas extrañas, regular el equilibrio hídrico y electrolítico, y también cumplir con una función regulatoria del equilibrio ácido-base.

Cada riñón de una persona adulta pesa 150 g y su tamaño es aproximadamente el de un puño. La unidad funcional del riñón es la nefrona. Un ser humano cuenta con aproximadamente de 800000 a 1000000 nefronas por cada riñón [1], cada una de las cuales está conformada por un glomérulo, que es un conjunto de capilares formados por las siguientes capas: el endotelio capilar, la membrana basal y una capa de células epiteliales. En conjunto, estas tres capas permiten el filtrado sanguíneo de tal forma que retienen proteínas plasmáticas y elementos formes, impidiendo su paso.

El filtrado que ha pasado por el glomérulo se dirige a la cápsula de Bowman, la cual rodea a este, y de ahí transita por un conjunto de túbulos renales (túbulo proximal, asa de Henle, túbulo distal, túbulo y conducto colectores) para dirigirse hacia la uretra por medio del uréter.

Mientras el filtrado glomerular pasa por los túbulos renales, ocurren dos procesos importantes: la reabsorción y la secreción. La primera hace referencia a que diversas sustancias necesarias para el cuerpo, como electrolitos, glucosa y aminoácidos, regresan al torrente sanguíneo, en cambio la segunda consiste en que algunas sustancias son secretadas desde la sangre hacia los túbulos renales con la finalidad de que estas sean expulsadas por medio de la orina, ya que son tóxicas para el cuerpo humano y suelen ser productos finales del metabolismo (urea, creatinina, ácido úrico, etc.) o bien es necesario eliminarlas para mantener un equilibrio hídrico y electrolítico ${ }^{[1]}$.

Cuando las funciones que debe llevar a cabo el riñón no se están realizando correctamente, puede haber alguna enfermedad renal y una de las más comunes es la enfermedad renal crónica (ERC), la cual es un gran problema en México y en su mayor parte es consecuencia de enfermedades crónicas no transmisibles mal atendidas, como el sobrepeso, la obesidad, la diabetes, la hipertensión arterial sistémica y dislipidemias, entre otras [2].
La guía del consorcio KDIGO (Kidney Disease: Improving Global Outcomes) define la ERC como anormalidades en la estructura o función del riñón, presente durante más de tres meses, con implicaciones para la salud. Cuando la tasa de filtración glomerular (TFG) es menor a $60 \mathrm{~mL} / \mathrm{min} / 1.73 \mathrm{~m}^{2}$ se considera ERC y se clasifica en cinco etapas con base en la TFG: si en la 5 la TFG es $<15 \mathrm{~mL} / \mathrm{min} / 1.73 \mathrm{~m}^{2}$, se considera como falla renal y es necesario utilizar una terapia de sustitución renal [3].

La terapia de sustitución renal incluye diálisis peritoneal, hemodiálisis y trasplante renal. Sin duda, este último es la mejor opción para tratar la ERC, sin embargo, en México no es tan viable debido al nivel de deterioro orgánico que presentan los pacientes por las enfermedades primarias, los altos costos iniciales y ante todo la falta de donaciones ${ }^{[4]}$.

Hasta julio de 2020, 17425 personas esperaban recibir un riñón y solamente se realizaron 641 trasplantes. Está claro que no ha sido posible cubrir esta demanda, pues tan solo en 2017 había 13634 personas esperando un donante y a pesar de que se realizaron trasplantes, aumentó la cifra de personas que requieren un riñón [5]. Por ello, es de suma importancia la implementación de nuevas alternativas que puedan sustituir la función renal, como los dispositivos médicos basados en órganos en chips (organs-on-chips, OOC).

Los OOC son dispositivos que se desarrollan utilizando técnicas de elaboración de microfluidos que contienen cámaras continuamente perfundidas, habitadas por células vivas colocadas para simular la fisiología a nivel de tejidos y órganos. Estos dispositivos producen múltiples niveles de funcionalidad de tejidos y órganos, que no son factibles con los sistemas de cultivo convencionales en $2 \mathrm{D}$ y $3 \mathrm{D}$.

Un dispositivo OOC ofrece una amplia gama de aplicaciones, como el modelado de enfermedades, la seguridad y eficacia de medicamentos y la medicina personalizada. Por lo tanto, es probable que a corto plazo lleguen a ser complementos o sustitutos de los métodos tradicionales de cultivo celular y de estudios en animales in vivo. Esta tecnología ha presentado avances dramáticos y en los últimos años ha sido propuesta por varios investigadores como plataforma para desarrollar dispositivos implantables que sustituyan la función de un órgano. 
En el presente trabajo se analiza el progreso en los diferentes modelos propuestos para la implementación de un microambiente que simule las condiciones fisiológicas renales y se enfoca, sobre todo, en los dispositivos microfluídicos de tipo organ-on-a-chip, cuyo enfoque es auxiliar o suplantar la función renal.

Un objetivo explícito de esta revisión es describir los avances de los dispositivos biomédicos de remplazo de la función renal por componentes del riñón y, como tal, se revisaron los resultados remarcables de cada componente por separado. Este estudio gira en torno a la recopilación y clasificación de estudios en los cuales se manifiesta la región del riñón a la que se dirige el desarrollo del dispositivo y en los que se menciona las líneas celulares empleadas. Finalmente, se hace hincapié en los dispositivos que están en fases clínicas.

\section{METODOLOGÍA}

Se realizó la búsqueda de artículos sobre dispositivos o modelos encaminados a la futura realización de un riñón artificial implantable, tales como dispositivos que incluyen cultivos de células renales, dispositivos microfluídicos (organ-on-a-chip de riñón), modelos impresos 3D o bien la combinación de diferentes técnicas.

La búsqueda de información se realizó utilizando palabras clave como kidney, device, chip, artificial, implantable, en Springer-Nature, Elsevier, Web of Science y Royal Society of Chemistry.

La recopilación de información se realizó por años, iniciando por los artículos publicados en 2010 y terminando en los publicados en 2020. La búsqueda finalizó en agosto de 2020 y se recopiló un total de 59 artículos, de los cuales se descartaron 27 debido a que trataban temas como la comprobación de la función de un túbulo proximal desde el punto de vista físico o bien eran trabajos de revisión (reviews) que proporcionaban información generalizada acerca del tema. Posteriormente se clasificó la información obtenida, de acuerdo con la parte del riñón a la que estaba dirigido cada estudio.

\section{RESULTADOS Y DISCUSIÓN}

A continuación se presentan los resultados de este trabajo, en donde se mencionan los trabajos de diversos autores. Cabe señalar que todos los artículos mencionados son sintetizados al final en la Tabla 1.

\section{Túbulos renales}

Jang et al. [6] desarrollaron un dispositivo microfluídico multicapa (MMD) para el eficiente cultivo y análisis de células tubulares renales, que está constituido por un canal de polidimetilsiloxano (PDMS), una membrana a base de poliéster y un depósito de PDMS unidos con un tratamiento de plasma.

Este dispositivo cuenta con un canal superior que provee un área de luz, la región de la membrana apical de células es expuesta a dicha área con un fluido tubular interno (fluido precursor de la orina). También cuenta con un canal inferior que provee un área intersticial, la cual está en contacto con la región de la membrana basolateral con un fluido tubular externo (torrente sanguíneo) para la estimulación e intercambio.

El MMD se conectó a una bomba de jeringa con tubos de silicona para aplicar un fluido de cizallamiento en las células cultivadas y posteriormente se colocó sobre un plato de cultivo que contenía fluido tubular externo para proveer suficiente circulación de aire y estimulación hormonal. En este MMD se utilizaron células primarias de conducto colector medular interno de rata (IMCD) y se encontró que la aplicación de una tensión de cizallamiento fluídico de $1 \mathrm{dyn} / \mathrm{cm}^{2}$ durante 5 horas y el utilizar la membrana porosa permitió condiciones fluídicas óptimas para las células cultivadas. Esto se verificó por medio de una mayor polarización celular, reorganización citoesquelética y transporte molecular mediante estimulaciones hormonales.

De manera similar, Ferrell et al. ${ }^{[7]}$ implementaron un biorreactor para evaluar células epiteliales de riñón bajo condiciones fisiológicas relevantes de flujo. El dispositivo implementado consta de cámaras de fluidos apicales y basolaterales, conectadas por medio de una membrana microporosa transparente.

La cámara superior contiene canales microfluídicos para perfundir la superficie apical de las células y la inferior actúa como un depósito para el transporte a través de la capa celular y proporciona soporte para la membrana. Los electrodos de resistencia eléctrica transepitelial (TEER) fueron integrados en el dispositivo para moni- 
M. B. García-San Luis y C. Chapa-González. Avances de la Tecnología Organ-on-achip de Riñón hacia Dispositivos de Remplazo de la Función Renal

ARTÍCULO DE REVISIÓN

torear el crecimiento celular y evaluar la integridad de las uniones estrechas entre célula-célula.

Se utilizaron células epiteliales de riñón humanas (HREC) y caninas Madin-Darby (MDCK) que fueron cultivadas en el biorreactor por perfusión. Además, se demostró una función diferenciada (formación de cilios y de uniones apretadas) en ambos tipos de células. Se observaron también cambios fenotípicos (reorganización citoesquelética de F-actina) en células humanas al someterlas a un esfuerzo cortante. El sistema TEER fue usado para evaluar cómo se formaba la monocapa polarizada de células y también se utilizó para medir el paso de inulina después de la ruptura de uniones estrechas por interruptor de $\mathrm{Ca}^{2+}$. Los resultados anteriores indicaron que este sistema experimental puede ser utilizado para estudiar procesos dependientes de flujo en células epiteliales renales.

Por otro lado, Sciancalepore et al. ${ }^{[8]}$ desarrollaron un dispositivo bioartificial de túbulo renal (organ-on- $a$ chip) que se compone de dos capas elastoméricas superpuestas, en medio de las cuales se encuentra una membrana porosa de policarbonato. La geometría fue propuesta para simular la estructura del túbulo renal, con un microcanal superior que provee el área de luz, en la que la porción apical de las células progenitoras del túbulo renal adultas (ARPC) estaba expuesta y un microcanal inferior simulando el área intersticial en contacto con las membranas basolaterales. Exponer a las células a una tensión de corte fluídica permitió que el transportador acuaporina-2 se localizara en la región apical y la bomba $\mathrm{Na}+\mathrm{K}+\mathrm{ATPasa}$ en la porción basolateral, en contraste con las ARPC cultivadas estáticamente. Con el dispositivo se obtuvo una recuperación de urea de $20 \pm 5 \%$ y de creatinina de $13 \pm 5 \%$.

Sateesh et al. ${ }^{[9]}$ diseñaron un modelo microfluídico de kidney-on-a-chip, en el que se analizó la tensión de corte fluídica en relación con la temperatura. Este dispositivo tiene una estructura en forma de $\mathrm{T}$ y consta de una entrada localizada en el fondo, con salidas ubicadas en las caras de las extremidades. Además, cuenta con un canal en el que hay un espacio rectangular designado para cultivar células renales. La longitud del canal intermedio regula la TFG (trabaja como una retroalimentación glomerular), ralentiza el líquido y aumenta la velocidad para que la presión sea tan baja como sea posible. La reacción se lleva a cabo en los sitios de cultivo de células renales, los solutos reaccionados y el fluido de
Cultura Científica y Tecnológica • Vol. 18, no. 1

Págs. 1-14 • Enero-Abril 2021

la luz sin reaccionar se separa en dos canales que son dirigidos a las arterias sanguíneas y el tracto urinario respectivamente. Con ello la temperatura tuvo una relación inversa con la tensión de corte fluídica, a una temperatura absoluta $(273.15 \mathrm{~K})$ la presión fue de $9.7 \mathrm{~Pa}$ y alcanzó $3.77 \mathrm{~Pa}$ a la temperatura corporal (308.65 K), mostrando un decremento en la presión. Este estudio demuestra el efecto de la temperatura sobre la tensión de corte fluídica.

Sin embargo, los estudios empleando sistemas microfluídicos continuaron. Guha et al. [10] utilizaron un modelo de kidney-on-a-chip en el cual se estudió la reabsorción dependiente de tamaño en túbulos renales, considerando las implicaciones de presión hidrostática. Dicho dispositivo se compone de dos túbulos sanguíneos separados por un túbulo principal en el centro, el cual conecta ambos mediante canales de transporte.

Los canales de transporte permiten el flujo de solutos desde el túbulo principal a los túbulos sanguíneos, dependiendo del tamaño de los solutos. Para analizar los efectos hidrostáticos, se emplearon obstáculos en forma de barras colocadas a manera de zigzag en el túbulo principal, formando una estructura escalonada. Los resultados mostraron una velocidad de salida de flujo de $9.12 \times 10^{-5}$ $\mathrm{m} / \mathrm{s}$, en comparación con $8.12 \times 10^{-5} \mathrm{~m} / \mathrm{s}$ sin efecto hidrostático. La tasa de reabsorción se mejoró de 50 a 55\%.

En otra investigación sobre el intercambio de fluidos en los túbulos renales, Huang et al. [11] estudiaron la funcionalidad de las células epiteliales renales con constitución tipo cápsula, en un dispositivo microfluídico de cocultivo. Se utilizaron células MDCK y células madre derivadas de tejido adiposo encapsuladas en gel de colágeno (CG-ASC). En este sistema de cocultivo, las dos clases de células se cultivaron dentro de canales microfluídicos, individualmente. El dispositivo microfluídico desarrollado proporcionó células madre de tejido adiposo encapsuladas, integradas en el gel de colágeno, y estas interactuaron directamente con las MDCK.

El canal periférico en forma de copa permitió que las MDCK tuvieran una interfaz grande con el canal central que contenía CG-ASC. El cocultivo con GC-ASC mejoró el desarrollo de cilios y las funciones epiteliales renales en las MDCK. Se confirmó que la plataforma de cocultivo puede aplicar el esfuerzo cortante en las células epiteliales y permitir que se lleve a cabo el intercambio de fluidos. 
De manera similar, Brakeman et al. [12] desarrollaron un sistema microfluídico para evaluar células renales epiteliales polarizadas bajo condiciones de flujo de fluidos relevantes. Los autores emplearon células HREC primarias, el soporte celular para el biorreactor se basó en una membrana microporosa disponible en un formato de seis pozos, permitiendo que las células fueran sembradas. El biorreactor estaba conformado por un camino microfluídico apical y una cámara basolateral estática que es fácilmente accesible desde el exterior del dispositivo.

El uso de este sistema permitió demostrar que las células pueden ser monitoreadas rutinariamente para observar la integridad de la monocapa (fuga de inulina) y la polarización, previo a la aplicación de fuerza cortante. También quedó de manifiesto que la habilidad de perfundir y recuperar con rapidez células completamente diferenciadas es adecuada para evaluar efectos a corto plazo del esfuerzo cortante en caminos de señalización inestables, tales como la fosforilación proteica y la presencia de citoquina mRNA.

Por otro lado, Shen et al. [13] decidieron centrar su estudio en el impacto de la curvatura de membranas de fibras huecas en células tubulares renales. Para ello utilizaron células de epitelio tubular proximal humano (HK-2) y MDCK, y a continuación prepararon varias fibras huecas con curvatura variable y membrana plana con curvatura cero. Las células renales en fibras huecas, aunque mostraron una morfología confluente como las de las membranas planas, expresaron funciones renales más altas y, además, estas funciones aumentaron significativamente con la curvatura de la membrana entre las fibras huecas. Con ello se determinó que al aumentar la curvatura del sustrato se podrían regular las funciones de las células sin alterar la morfología celular confluente.

Del mismo modo, Jansen et al. [14] emplearon células humanas epiteliales del túbulo proximal condicionalmente inmortalizadas (ciPTEC) para proponer un modelo de túbulo renal. En primer lugar, estudiaron el rol de los transportadores de aflujo y eflujo en la eliminación de toxinas urémicas en monocapas planas y, en segundo, desarrollaron cultivos 3D de células polarizadas de ciPTEC en membranas de fibra hueca de polietersulfona. Finalmente se confirmó el aclaramiento secretorio de indoxil sulfato de suero humano unido a albúmina y ácido quinurénico, así como la reabsorción de albúmina.
Para estudiar la reabsorción de urea, Jong et al. ${ }^{[15]}$ desarrollaron un quimiosorbente con una densidad alta de grupos de ninhidrina, capaces de unir covalentemente la urea bajo condiciones fisiológicas. Para ello se prepararon cuentas macroporosas por polimerización de suspensión de 5-vinil-1-indanona (vinilindanona) usando una mezcla 90:10 (v/v) de tolueno y nitrobenceno como porógeno.

Los grupos de indanona fueron posteriormente oxidados a grupos de ninhidrina. La cinética de absorción de urea se evaluó bajo condiciones estática y dinámica a $37^{\circ} \mathrm{C}$ en un dializado (urea en solución salina tamponada con fosfato). Bajo condiciones estáticas y a un cociente molar 1:1 de ninhidrina:urea, las cuentas sorbentes removieron $\approx 0.6-0.7 \mathrm{mmol}$ urea/g y bajo condiciones dinámicas y a un exceso molar 2:1 de ninhidrina removieron $\approx 0.6 \mathrm{mmol}$ urea/g sorbente en $8 \mathrm{~h}$ a $37^{\circ} \mathrm{C}$. Estos resultados indicaron que el quimiosorbente desarrollado es potencialmente adecuado para utilizarse en un riñón artificial portátil.

\section{Túbulo proximal}

Zhuang et al. [16] investigaron el transporte en túbulo proximal renal, para lo cual desarrollaron un túbulo utilizando células HK-2 primarias. Para diseñarlo, se utilizó una membrana de fibra hueca una capa exterior de piel que proporciona inmunoprotección para las células del flujo sanguíneo extracapilar y una superficie interna gruesa que facilita un recubrimiento de hidrogel para el acceso de la celda.

Dicha membrana se incrustó en un modelo de lab-on$a$-chip para la prueba exploratoria a pequeña escala bajo condiciones de flujo. La membrana de fibras huecas con acción reabsortiva se diseñó con una capa de piel en el espacio externo de la fibra, que podía rechazar sustancias indeseadas del flujo sanguíneo y minimizar la absorción proteica en la fibra. Su superficie interna tenía poros de hasta $0.3 \mu \mathrm{m}$, facilitando la fijación de la $\mathrm{cu}$ bierta de la matriz extracelular (ECM) sobre la membrana, junto con una cubierta de fibrina y una monocapa confluente de células HK-2.

Las células demostraron la polarización correcta para reabsorber los solutos deseados del filtrado glomerular de regreso a la sangre con funcionalidades de transporte. El uso de una membrana con acción reabsortiva de fibras huecas y recubrimiento de fibrina para un mejor 
soporte de las células renales, proporcionó características muy ventajosas para el desarrollo de un dispositivo renal bioartificial mejorado.

Posteriormente, Vriend et al. ${ }^{[17]}$ investigaron el rol de la tensión de fuerza fluídica y de los cilios primarios en la actividad de transporte y morfología en las células epiteliales de túbulo proximal (PTEC, por sus siglas en inglés) sembradas en un tubule-on-a-chip. Este dispositivo consta de un canal de gel y otro de perfusión. Al primero se puede accesar por una entrada específica y al segundo por la entrada del canal medio.

Las células cultivadas en el canal de perfusión en la interfaz del medio-ECM fueron expuestas a una tensión completa de corte fluídica y establecidas como la región de interés en este experimento. Como resultado, la tensión de corte fluídica aumentó la recepción de albúmina y el eflujo de glicoproteína-P y elongación celular. Esto no se atribuyó al mecanismo mecanosensitivo relacionado a los cilios primarios en PTEC, pero los autores indicaron que probablemente se deba a las microvellosidades presentes en la membrana apical.

Sakolish et al. [18] también utilizaron un dispositivo microfisiológico con PTEC para predecir, en este caso, la reabsorción renal de cinco componentes: creatinina (control negativo), ácido perfluorooctanoico (control positivo), cisplatino, gentamicina y cadmio. Para ello perfundieron un medio que contenía los compuestos ajustados para uniones no específicas con el fin de determinar la reabsorción renal. Además, para modelar la cinética de reabsorción y hacer predicciones del aclaramiento renal in vivo, utilizaron un modelo de tubo paralelo.

Este estudio demostró que el chip de túbulo proximal, cuando es combinado con un modelo fisiológico para el aclaramiento renal en general y un modelo físico para el túbulo, provee predicciones exactas para la reabsorción tubular, cualitativa y cuantitativamente. Como este dispositivo carece de un canal vascular, es más apropiado para predecir la cinética de componentes no secretados que son activamente reabsorbidos y acumulados en el riñón, como el cadmio.

También en el ámbito del estudio de la reabsorción renal, Lin et al. [19] desarrollaron modelos impresos 3D de túbulo proximal vascularizados, compuestos por conductos adyacentes linearizados con epitelio confluente y endotelio, incrustados en una ECM permeable e independientemente dirigida, usando un sistema de perfusión de bucle cerrado. Durante la perfusión a largo plazo, las PTEC dentro del epitelio confluente exhibieron una gran mejoría en su densidad y en la longitud de sus microvellosidades.

Cuando la albúmina marcada con fluorescencia y la inulina fueron perfundidas juntas a través del túbulo proximal, la albúmina fue selectivamente reabsorbida. Por último, bajo condiciones de hiperglicemia se demostró que los modelos diseñados proveen una plataforma única para explorar interacciones relevantes en enfermedades, tal como la diabetes.

Por su parte, Homan et al. [20] utilizaron un método para crear túbulos proximales humanos 3D in vitro, que combina bioimpresión, cultivo celular 3D y conceptos de organ-on-a-chip para crear un túbulo proximal complejo compuesto de una luz abierta perfusable con una arquitectura programable, la cual puede mantener la heterogeneidad celular extratubular.

Estos túbulos proximales consisten en una arquitectura de luz abierta circunscrita por PTEC que están completamente incrustados en una ECM y alojados en chips de tejido perfundible, en donde son sometidos a fuerzas de corte fisiológicas. El epitelio exhibió varias características morfológicas y marcadores funcionales similares a PTEC nativas in vivo. El túbulo proximal 3D permitió la colección de cientos de miles de células para su análisis, mucho mejor que las requeridas para un muestreo preciso por citometría de flujo. Los resultados mostraron morfología epitelial y propiedades funcionales relativamente mejores a las de modelos en 2D.

En otro estudio, Sateesh et al. [21] diseñaron una estructura para imitar la reabsorción dependiente de tamaño de las células endoteliales en el túbulo contorneado proximal, que resultó ser la primera matemáticamente modelada y posteriormente analizada. El diseño de dicha estructura es el siguiente: un tubo principal que lleva el fluido de la luz es colocado en el medio de dos túbulos portadores de sangre. Hay canales de transporte que conectan los túbulos sanguíneos a lo largo de ambos lados del túbulo principal. El canal de transporte permite que los solutos y las proteínas más pequeñas pasen a través del canal sin esfuerzo. Los dos lados del túbulo principal están diseñados con canales para mejorar la tasa de reabsorción. El ángulo entre el canal y 
el túbulo principal controla el flujo de fluido del túbulo contorneado proximal al túbulo sanguíneo, lo cual beneficia la reabsorción al colectar los solutos del túbulo principal en el sitio de entrega de sangre.

En cuanto a los resultados obtenidos, el canal recto mostró un flujo promedio uniforme de $4.77 \times 10-7 \mathrm{~m} / \mathrm{s}$ con una tasa de reabsorción del $46 \%$, mientras que el canal inclinado presentó una velocidad de flujo promedio de $4.77 \times 10^{-7} \mathrm{~m} / \mathrm{s}$ con una tasa de reabsorción del $52 \%$. Las observaciones probaron que el canal inclinado promueve mayor flujo, el cual mejora la tasa de reabsorción. El número de canales requeridos para compararse con la reabsorción de una nefrona nativa se calculó ser de 1000 por $3000 \mathrm{~nm}$ de longitud. Gracias a la complejidad del diseño, el incremento del número de canales mejoró la exactitud de la tasa de reabsorción.

\section{Glómerulo}

Ould-Dris et al. ${ }^{\text {[22] }}$ realizaron un microchip para el estudio del filtrado glomerular y de la transferencia de masa de urea, cobalamina (vitamina B12) y albúmina. Este dispositivo se compone de tres capas: dos de PDMS $y$, en medio de ellas, una de PES (polietersulfona). Cada capa PDMS incluye una puerta de entrada y una de salida, así como una cámara para poder filtrar. Una matriz de microcanal de $100 \mu \mathrm{m}$ de profundidad, localizada en el fondo de cada cámara, se agregó para crear un tamiz diseñado para imposibilitar el bloqueo de la filtración por la deformación de la membrana durante la perfusión fluídica.

En cada capa de PDMS, los puertos de entrada y salida están perforados, lo cual dio como resultado dos circuitos microfluídicos separados. Un conjunto de microcanales de entrada y salida se usó para conectar la cámara de filtración y los puertos de entrada y salida. En este estudio, la introducción del tamiz dentro del microchip permitió controlar los procesos de transferencia de masa, ya sea controlando la presión en la cámara de filtración o la porosidad de la membrana.

Petrosyan et al. ${ }^{[23]}$ decidieron enfocarse en el estudio de la barrera de filtración glomerular, para lo cual utilizaron un glomerulus-on-a-chip que está constituido por podocitos humanos y células endoteliales glomerulares (GEnC) humanas sembradas en organoplatos y se caracteriza por no tener una membrana artificial que separe a sus dos monocapas.
En este estudio, las células del glomérulo pudieron interactuar para generar una capa de matriz extracelular compuesta por colágeno IV y laminina. En cultivos a largo plazo, las células mantuvieron su morfología, formaron estructuras similares a capilares y expresaron proteínas de diafragma cortadas. Cuando los chips se expusieron a suero de pacientes con autoanticuerpos antipodocitos, hubo albuminuria proporcional a la proteinuria de los mismos pacientes, un fenómeno no observado en el suero de controles sanos o individuos con defectos en los podocitos primarios.

Los resultados citados del párrafo anterior muestran su aplicación en modelos de enfermedad renal y desarrollo de fármacos, como en el trabajo realizado por Zhou et al. ${ }^{[24]}$, ya que estudiaron la nefropatía hipertensiva en un glomérulo on-a-chip, para lo cual se utilizaron GEnC inmortalizadas y células indiferenciadas MPC5 (clon de podocitos de ratón). Este dispositivo usa canales de PDMS compartimentados para lograr la formación de una barrera de filtración glomerular en una membrana cubierta con ECM (matriz extracelular).

También se suplementó perfusión de flujo al microcanal superior y se provocaron fuerzas mecánicas (presión capilar glomerular y fuerza de corte) para actuar en la membrana de la barrera de filtración glomerular. Dieciséis cámaras de cultivo integradas on-a-chip fueron compuestas de capas: inferiores y superiores de PDMS y una intermedia de policarbonato. Tres capas fueron alineadas e irreversiblemente unidas para formar dos sets de microcanales separados por medio de una membrana porosa, conteniendo un arreglo de agujeros pasantes con un diámetro igual a $10 \mu \mathrm{m}$.

Gracias al glomérulo on-a-chip se demostró que las fuerzas glomerulares mecánicas tienen un rol muy importante en lo que respecta al rearreglo citoesquelético celular, así como en el daño en las células y sus uniones que provocan un incremento en la fuga glomerular observada en la nefropatía hipertensiva. La hipertensión glomerular dañó el endotelio y las uniones intercelulares en el glomérulo. Este dispositivo permitió simular el microentorno hemodinámico en el glomérulo en condiciones fisiológicas, cuando se usa una tasa de flujo de $5 \mu \mathrm{L} / \mathrm{min}$ en los canales endoteliales. El chip glomerular pudo filtrar la mayoría de las moléculas de inulina y pocas de albúmina, por lo que estos resultados sugirieron una filtración alta comparada con los modelos in vivo. 
También se estudió la nefrotoxicidad inducida por drogas. Li et al. ${ }^{[25]}$ utilizaron un dispositivo microfluídico compartimentado para investigar la nefrotoxicidad inducida por cadmio. El dispositivo, fabricado con PDMS, contenía tres canales: uno de cultivo celular, uno de gel y uno colector. El canal central fue perfundido con colágeno I para soportar la adhesión celular y el crecimiento en la matriz 3D. El canal de cultivo fue sembrado con GEnC primarias de rata para simular la parte capilar de la barrera de filtración glomerular. El canal colector fue usado para representar la cápsula glomerular y colectar las sustancias filtradas del canal de cultivo mediante la matriz. Las GEnC se sometieron a diferentes concentraciones de cadmio.

Los resultados mostraron morfología celular anormal, viabilidad celular reducida, uniones célula-célula interrumpidas y mayor permeabilidad en GEnC en presencia de cadmio, lo que coincidió con las respuestas inducidas in vivo.

Por otro lado, Slater et al. [26] desarrollaron dos modelos de una tricapa de la pared capilar glomerular. El primero se basó en insertos de cultivo de tejidos y proporcionó evidencia de interacción célula-célula a través de mediadores solubles y en el segundo, el soporte sintético del inserto de cultivo de tejidos se remplazó con una nueva membrana bioartificial compuesta que consistió en una membrana de nanofibras que contenía colágeno I, electrohilada directamente sobre una malla de soporte de níquel fino micro-fotoelectroformado. Las GEnC y los podocitos crecieron en monocapas a ambos lados del soporte del inserto para formar un modelo constituido por tres capas que simuló el capilar glomerular humano in vitro.

Hacia un modelo mejorado, Musah et al. ${ }^{\text {[27] describie- }}$ ron un protocolo detallado para la diferenciación directa de células madre pluripotentes inducidas humanas (hiPS) a podocitos maduros renales y el establecimiento de un glomerulus-on-a-chip. Primero, tras múltiples pasos se diferenciaron las hiPS en células del mesodermo intermedio y, después de obtenerlas, se pueden realizar dos diferentes metodologías: la primera, que consiste en la generación de podocitos utilizando platos de cultivo, y la segunda, que se caracteriza por generar podocitos utilizando un dispositivo microfluídico organ-on-a-chip.
Tanto la diferenciación de podocitos como el cocultivo con células primarias endoteliales glomerulares en lados opuestos de la membrana de PDMS en el dispositivo microfluídico permitieron establecer el glomerulus-on-a-chip, en el cual, los podocitos interconectados y las capas de células endoteliales expresaron nefrina y cadherina-Ve, respectivamente. En conjunto, este protocolo proporcionó un método químicamente definido para la derivación de podocitos maduros que recapitulan la estructura y función de la pared capilar glomerular en un organ-on-a-chip. Además de que puede ayudar al estudio de la toxicidad inducida por fármacos, este modelo microfluídico también puede ser usado para estudiar la filtración molecular selectiva y posteriormente podría utilizarse en bioimpresión 3D de órganos, ingeniería tisular o en medicina regenerativa.

Hacia el desarrollo de un dispositivo implantable, Kensinger et al. ${ }^{[28]}$ implementaron una membrana con poros con una singular forma de hendidura en un cartucho de hemofiltrado -implantado en perros de clase A- para optimizar la compensación entre la permeabilidad y selectividad. El funcionamiento del cartucho es el siguiente: la sangre se mecaniza hacia el fondo, el cual recibe la membrana nanoporosa de silicona y es retenida por una tapa que drena el ultrafiltrado; esta membrana es sellada al cartucho por juntas, el cual, a su vez, es conectado a la aorta y a la vena cava por injertos de politetrafluoroetileno y, finalmente, el líquido proveniente de los filtros de silicona es drenado a las bolsas de colección.

Una de las principales ventajas de este dispositivo es la operación libre de bomba, ya que disminuye de manera generalizada el tamaño del dispositivo implantable y aumenta la probabilidad de éxito de operación a largo plazo. El cartucho de hemofiltración tiene la habilidad de operar sin sistemas de coagulación a largo plazo (solo se utilizó aspirina como anticoagulante). Las tasas de ultrafiltrado y tamaños de poro calculados de la permeabilidad hidráulica observada, en general coincidieron con las metas de diseño y los coeficientes de tamizado de albúmina y polisacáridos globulares.

\section{Nefrona}

Para imitar una nefrona, $\mathrm{Mu}$ et al. ${ }^{[29]}$ realizaron una conjugación de fibrilogénesis y moldeo de líquidos para así poder diseñar una red vascular $3 \mathrm{D}$ en hidrogel. Se 
utilizó las siguientes clases de células: endoteliales de la vena umbilical humana primaria (pHUVEC), MDCK, de fibroblastos NIH 3T3 y las THP-1. Esta técnica permitió la imitación de la difusión pasiva en una nefrona y se demostró que la cobertura celular en los microcanales de hidrogel tiene el potencial de simular la transferencia de masa.

Un chip microfluídico de tres capas para evaluar la nefrotoxicidad inducida por fármacos fue desarrollado por Yin et al. [30], el cual incluye dos partes: 1) generador del gradiente de concentración de fármaco microfluídico y 2) una plataforma de temperatura controlada para el cultivo de células renales. En este estudio, las RPTEC y las células endoteliales capilares peritubulares fueron cubiertas con los fármacos y, a diferencia de las células cultivadas en placas petri, las cultivadas en el dispositivo mostraron un rendimiento mayor en términos de crecimiento celular y evaluación de la nefrotoxicidad de fármacos.

Un significativo decremento en la nefrotoxicidad inducida por cisplatino fue encontrada debido a la intervención de cimedicina en el dispositivo, por tanto, este puede facilitar el desarrollo de fármacos.

Por su parte, Kim et al. [31] utilizaron un kidney-on-achip para comparar la nefrotoxicidad de la gentamicina, administrada en una misma dosis, pero utilizando diferentes regímenes farmacocinéticos. El dispositivo se compone de dos canales de PDMS: superior e inferior y en medio de ambos una membrana porosa de poliéster. Se probaron dos regímenes de tratamiento de gentamicina con una misma dosis durante 24 horas. En un régimen se simuló los perfiles de aclaramiento de fármacos para inyección de bolo exponiendo las HREC a $19.2 \mathrm{mM}$ de gentamicina y reduciendo el nivel de dosis a la mitad cada 2 horas durante 24 horas. En el otro régimen se suministró gentamicina ( $3 \mathrm{mM}$ por 24 horas).

La inmunorreactividad de las uniones proteicas fue disminuida en ambos regímenes. La proteína $\mathrm{ZO} 1$ y la fluorescencia de ocludina disminuyó menos con el régimen que simula la inyección en bolo, el cual demostró menor citotoxicidad y permitió que el epitelio mantuviera una baja permeabilidad, mientras la infusión continua permitió un incremento en la citotoxicidad y permeabilidad. Estos datos demostraron que la gentamicina interrumpe las uniones célula-célula, aumenta la permeabilidad de la membrana y disminuye la viabi- lidad celular particularmente con exposición prolongada de nivel bajo, por lo tanto, se demostró el potencial general de usar modelos de cultivo microfluídicos.

\section{Dispositivos en fases clínicas}

En 2008 se realizó una prueba controlada en fase II, de etiqueta abierta, utilizando un dispositivo extracorpóreo de asistencia de túbulo renal (RAD) combinado con hemofiltración venovenosa continua $(\mathrm{CVVH}){ }^{[32]}$, en el que primero la sangre es filtrada por el circuito de CVVH y, posteriormente, por medio de una bomba peristáltica la sangre es bombeada hacia el espacio extracorpóreo del RAD, a una velocidad de $150 \mathrm{~mL} / \mathrm{min}$. El ultrafiltrado (UF) es dirigido hacia las fibras dentro del RAD a una velocidad de $10 \mathrm{~mL} / \mathrm{min}$, donde se encuentran células del túbulo renal humano no autólogas sembradas en la superficie interna, ajustándose ahí la presión hidráulica para poder realizar la reabsorción.

El tamaño de los poros de las fibras huecas y aunado a que no son biodegradables, permite que las fibras actúen como un andamio y a su vez como una barrera de inmunoprotección para las células. El UF que sale del espacio luminal del RAD es colectado y desechado como orina. La sangre que sale del RAD viaja a través de una bomba adicional y es enviada de vuelta al paciente. Este dispositivo, en conjunto con la CVVH, se utilizó en pruebas clínicas con 58 pacientes que padecían falla renal aguda, de los cuales un grupo de 18 solamente utilizaron terapia de remplazo renal continua. En el día 28 , la tasa de mortalidad fue de $33 \%$ en el grupo donde se utilizó RAD y de $61 \%$ en el grupo de pacientes asistidos solamente con terapia de remplazo renal continua. El análisis de Kaplan-Meier reveló que para el día 180 la supervivencia se vería mejorada de manera significativa. La terapia con RAD fue bien tolerada por los pacientes y también se asoció con una recuperación más rápida de la función renal [33].

En 2016 se llevó a cabo una prueba en humanos aprobada por la Administración de Medicamentos y Alimentos (FDA, por sus siglas en inglés), en la que utilizaron un riñón artificial portátil (WAK) basado en tecnología de sorbente regenerador de dializado ${ }^{[34]}$. Antes del tratamiento con WAK, cada participante se trató con una sesión de cuatro horas de hemodiálisis convencional. Después de dos horas sin ningún tratamiento, los pacientes fueron conectados al WAK, que pesa $5 \mathrm{~kg}$ y consiste en un sistema de hemodiálisis miniaturizado a 
base de sorbentes que utiliza un dializador comercialmente disponible, con un área superficial de $0.6 \mathrm{~m}^{2} \mathrm{y}$ un volumen de $52 \mathrm{~mL}$ en el compartimento sanguíneo. Por medio de una batería recargable se hace funcionar una bomba pulsátil con circuitos separados de doble canal que impulsan la sangre y es posteriormente dializada. El dializado es regenerado usando tres cartuchos con sorbente conectados en serie que contienen ureasa, fosfato de circonio, óxido de circonio hidratado y carbón activado.

Este sistema utiliza ureasa para convertir la urea en amoníaco y carbonato, los cuales, en presencia de iones hidrógeno, forman dióxido de carbono. Posteriormente, el amoníaco es adsorbido por el fosfato de circonio con otros cationes como $\mathrm{Ca}^{2+}, \mathrm{Mg}^{+}$y K $\mathrm{K}^{+}$, a cambio de $\mathrm{Na}^{+}$y $\mathrm{H}^{+}$. El dióxido de carbono es expulsado hacia la atmósfera como un gas mediante un sistema de desgasificación semipermeable removedor de burbujas. La capa de óxido de circonio hidratado remueve metales pesados, como cobre y plomo y también adsorbe aniones como fosfatos a cambio de acetato. Otros solutos de tamaño intermedio y componentes orgánicos son retirados por el carbono activado en la última capa.

El volumen total aproximado del circuito sanguíneo es de $65 \mathrm{~mL}$ y el del circuito de dializado es de $375 \mathrm{~mL}$. Adicionalmente, la ultrafiltración es controlada en el circuito dializador por microbombas, la infusión de heparina, acetato de calcio, acetato de magnesio y bicarbonato de sodio. Un detector ultrasónico de burbujas en la tubería de sangre arterial y un detector óptico de fugas sanguíneas en el tubo de dializado dispara una alarma audible y detiene la bomba principal.

En cuanto a los resultados de esta prueba, los pacientes tuvieron estabilidad hemodinámica, no hubo efectos adversos serios, el tratamiento fue bien tolerado y se llevó a cabo el aclaramiento de solutos urémicos de manera efectiva. Los electrolitos se mantuvieron estables y hubo homeostasis de fluidos. Cinco pacientes de los siete completaron el tratamiento de 24 horas. La prueba se detuvo después del séptimo sujeto debido a problemas técnicos, entre los cuales se destaca la presencia excesiva de burbujas de dióxido de carbono en el circuito de dializado [34].

En vías de desarrollo se encuentra un riñón artificial implantable que combina la tecnología de silicio con ingeniería tisular [35]. El dispositivo está en pruebas preclínicas y su función es imitar la fisiología de la nefrona, utilizando un hemocartucho combinado con un biocartucho que consiste en un biorreactor con células epiteliales de túbulo renal cultivadas. La sangre es procesada en el hemocartucho que contiene microchips hechos con tecnología de silicio, cada uno de los cuales tiene poros en forma de hendidura y en conjunto estos crean una membrana compacta porosa que imita al glomérulo al retener proteínas plasmáticas mientras deja pasar productos de desecho y electrolitos al biocartucho. Este regresa electrolitos, agua y glucosa a la sangre y concentra los productos de desecho en un líquido similar a la orina.

El dispositivo propuesto tiene una gran ventaja al no utilizar bombas eléctricas, ya que es directamente conectado al paciente. También evita el uso de un dializado, pues la reabsorción realizada en el biocartucho mantiene un balance fluídico neutral mientras se realiza la excreción de desechos. Los resultados de las pruebas preclínicas de este dispositivo aún no son reportados, sin embargo, la propuesta del dispositivo ya es un gran paso hacia la creación del riñón artificial implantable.

En general, en los tres estudios previamente mencionados se utilizaron conceptos básicos estudiados en trabajos anteriores, como son la implementación de sorbentes para el aclaramiento de solutos, el uso de una membrana porosa que imite la función del glomérulo y el cultivo de células renales, entre otros. Esto demuestra que es necesario el estudio previo de los componentes de cada dispositivo para lograr imitar la función renal.

\section{CONCLUSIONES}

La recopilación de la información en bases de datos de artículos científicos se logró empleando los criterios de búsqueda kidney, device, chip, artificial, implantable, por lo que fue posible resumir la información publicada y distinguir los avances de dispositivos biomédicos para el remplazo de la función renal. Algunos de los dispositivos propuestos hasta la fecha contienen células suspendidas o adheridas en una superficie confinada, por tanto, la caracterización del rendimiento de un proceso de filtración también requiere la consideración de indicadores biológicos como la viabilidad celular, las tasas de proliferación celular y la diferenciación o maduración celular adecuada. 
Imitar el entorno de los componentes del riñón y evaluar las funciones como la TFG en relación con las variables del diseño de los dispositivos es una propuesta compleja que solo algunos grupos de investigación en el mundo han explorado.

A partir de ello, se puede resaltar tres desafíos para avanzar en dispositivos que suplan la función renal: a) conocer la relación de la viabilidad y diferenciación celular con los procedimientos actuales de desarrollo de los organs-on-a-chip; b) estudiar el control de la reabsorción renal analizando la respuesta ante estímulos hormonales y c) implementar más modelos que imiten en conjunto la filtración y reabsorción renal.
Además, la heterogeneidad entre los resultados de las publicaciones revisadas, en términos del funcionamiento de cada dispositivo y la línea celular empleada dependiendo de la parte del riñón a la que se enfoca su estudio y el posible sesgo de publicación -derivado de que no todos los experimentos, desarrollos de dispositivos o ensayos clínicos realmente realizados hayan sido publicados por resultados negativos o no esperados-, deja ver que la maduración de esta tecnología emergente requiere aún de la generación de la evidencia científica suficiente que represente un avance hacia dispositivos traslacionales.

TABLA 1

Clasificación de los Dispositivos con Base en Cada Parte del Riñón

\begin{tabular}{|c|c|c|c|}
\hline $\begin{array}{l}\text { PARTE DEL } \\
\text { RIÑóN }\end{array}$ & Dispositivo o ENFOQUE DEL ESTUdio & LÍNEA CELULAR & ReF. \\
\hline \multirow[t]{10}{*}{$\begin{array}{l}\text { Túbulos } \\
\text { renales }\end{array}$} & $\begin{array}{l}\text { Dispositivo microfluídico multicapa para un eficiente cultivo y análi- } \\
\text { sis de células tubulares renales. }\end{array}$ & $\begin{array}{l}\text { Células primarias del conducto colector me- } \\
\text { dular interno de rata. }\end{array}$ & [6] \\
\hline & $\begin{array}{l}\text { Análisis de células epiteliales renales bajo condiciones fisiológicas re- } \\
\text { levantes de flujo. }\end{array}$ & $\begin{array}{l}\text { Células epiteliales renales humanas. } \\
\text { Células MDCK. }\end{array}$ & [7] \\
\hline & $\begin{array}{l}\text { Impacto de la curvatura de membranas de fibras huecas en células tu- } \\
\text { bulares renales. }\end{array}$ & $\begin{array}{l}\text { Células HK-2. } \\
\text { Células MDCK. }\end{array}$ & [13] \\
\hline & Dispositivo bioartificial de túbulo renal (organ-on-a-chip). & Células progenitoras del túbulo renal adultas. & [8] \\
\hline & $\begin{array}{l}\text { Túbulos renales 3D creados con bioingeniería para aclarar toxinas } \\
\text { urémicas. }\end{array}$ & $\begin{array}{l}\text { Células humanas epiteliales del túbulo proxi- } \\
\text { mal condicionalmente inmortalizadas. }\end{array}$ & {$[14]$} \\
\hline & $\begin{array}{l}\text { Modelo microfluídico de kidney-on-a-chip y análisis de la tensión de } \\
\text { corte fluídica en función del efecto de la temperatura. }\end{array}$ & Células renales. & [9] \\
\hline & $\begin{array}{l}\text { Modelo de kidney-on-a-chip en donde se estudia la reabsorción de- } \\
\text { pendiente de tamaño, considerando las implicaciones de presión hi- } \\
\text { drostática. }\end{array}$ & N/A & [10] \\
\hline & $\begin{array}{l}\text { Funcionalidad de las células epiteliales renales con constitución tipo } \\
\text { cápsula en un dispositivo microfluídico de cocultivo. }\end{array}$ & $\begin{array}{l}\text { Células MDCK. } \\
\text { Células madre derivadas de tejido adiposo } \\
\text { encapsuladas en gel de colágeno. }\end{array}$ & {$[11]$} \\
\hline & $\begin{array}{l}\text { Sistema microfluídico para evaluar células renales epiteliales polariza- } \\
\text { das bajo condiciones de flujo relevantes. }\end{array}$ & $\begin{array}{l}\text { Células epiteliales primarias renales huma- } \\
\text { nas. }\end{array}$ & {$[12]$} \\
\hline & $\begin{array}{l}\text { Quimiosorbente con una densidad alta de grupos de ninhidrina, ca- } \\
\text { paz de unir covalentemente la urea bajo condiciones fisiológicas y así } \\
\text { ser potencialmente adecuado para utilizarse en un riñón artificial por- } \\
\text { tátil. }\end{array}$ & $\mathrm{N} / \mathrm{A}$ & [15] \\
\hline \multirow[t]{4}{*}{$\begin{array}{l}\text { Túbulo } \\
\text { proximal }\end{array}$} & Transporte en túbulo proximal renal. & $\begin{array}{l}\text { Células epiteliales primarias de túbulo proxi- } \\
\text { mal humanas }\end{array}$ & [16] \\
\hline & $\begin{array}{l}\text { Método de bioimpresión para crear túbulos renales humanos 3D in } \\
\text { vitro. }\end{array}$ & Células epiteliales de túbulo proximal. & [20] \\
\hline & Modelos impresos 3D de túbulo proximal vascularizados. & $\begin{array}{l}\text { Células humanas epiteliales de túbulo proxi- } \\
\text { mal inmortalizadas. }\end{array}$ & [19] \\
\hline & $\begin{array}{l}\text { Estudio de la tensión de fuerza fluídica y de los cilios primarios en } \\
\text { la actividad de transporte y morfología en las células endoteliales de } \\
\text { túbulo proximal sembradas en un tubule-on-a-chip. }\end{array}$ & $\begin{array}{l}\text { Células humanas epiteliales del túbulo proxi- } \\
\text { mal condicionalmente inmortalizadas. }\end{array}$ & {$[17]$} \\
\hline
\end{tabular}


TABLA 1 (CONT.)

Clasificación de los Dispositivos con Base en Cada Parte del Riñón

\begin{tabular}{|c|c|c|c|}
\hline $\begin{array}{l}\text { PARTE DEL } \\
\text { RIÑóN }\end{array}$ & DisPositivo O ENFOQUE DEL ESTUDIO & LÍNEA CELULAR & REF. \\
\hline \multirow[t]{2}{*}{$\begin{array}{l}\text { Túbulo } \\
\text { proximal }\end{array}$} & $\begin{array}{l}\text { Estructura propuesta para imitar la reabsorción dependiente de ta- } \\
\text { maño de las células endoteliales en el túbulo contorneado proximal. }\end{array}$ & $\mathrm{N} / \mathrm{A}$ & [21] \\
\hline & $\begin{array}{l}\text { Dispositivo microfisiológico de túbulo proximal renal para predecir } \\
\text { la reabsorción renal de cinco componentes; creatinina (control nega- } \\
\text { tivo), ácido perfluorooctanoico (control positivo), cisplatino, genta- } \\
\text { micina y cadmio. }\end{array}$ & $\begin{array}{l}\text { Células primarias humanas de túbulo proxi- } \\
\text { mal renal. }\end{array}$ & {$[18]$} \\
\hline \multirow[t]{7}{*}{ Glomérulo } & $\begin{array}{l}\text { Filtración glomerular y la transferencia de masa de urea, cobalamina } \\
\text { (vitamina B12) y albúmina. }\end{array}$ & N/A & {$[22]$} \\
\hline & Filtración glomerular. & $\begin{array}{l}\text { Células endoteliales glomerulares. } \\
\text { Podocitos. }\end{array}$ & [26] \\
\hline & Estudio de la nefropatía hipertensiva en un glomerulus-on-a-chip. & $\begin{array}{l}\text { Células endoteliales glomerulares inmorta- } \\
\text { lizadas. } \\
\text { Células indiferenciadas MPC5. }\end{array}$ & [24] \\
\hline & $\begin{array}{l}\text { Dispositivo microfluídico compartimentado para investigar la nefro- } \\
\text { toxicidad inducida por cadmio. }\end{array}$ & $\begin{array}{l}\text { Células primarias endoteliales glomerulares } \\
\text { de rata. }\end{array}$ & {$[25]$} \\
\hline & $\begin{array}{l}\text { Descripción de un protocolo detallado para la diferenciación directa } \\
\text { de células madre pluripotentes inducidas a podocitos maduros renales } \\
\text { y el establecimiento de un glomerulus-on-a-chip. }\end{array}$ & $\begin{array}{l}\text { Células madre pluripotentes inducidas hu- } \\
\text { manas. }\end{array}$ & {$[27]$} \\
\hline & Glomerulus-on-a-chip para simular la barrera de filtración glomerular. & $\begin{array}{l}\text { Podocitos humanos. } \\
\text { Células endoteliales glomerulares. }\end{array}$ & {$[23]$} \\
\hline & $\begin{array}{l}\text { Membrana con poros en forma de hendidura para optimizar la com- } \\
\text { pensación entre la permeabilidad y selectividad, permitiendo la tera- } \\
\text { pia implantada. }\end{array}$ & N/A & [28] \\
\hline \multirow[t]{3}{*}{ Nefrona } & Diseño de una red vascular 3D en hidrogel para imitar una nefrona. & $\begin{array}{l}\text { Células endoteliales de la vena umbilical hu- } \\
\text { mana primaria. } \\
\text { Células MDCK. } \\
\text { Células de fibroblastos NIH 3T3. } \\
\text { Células THP-1. }\end{array}$ & [29] \\
\hline & $\begin{array}{l}\text { Chip microfluídico de tres capas, el cual provee un ambiente simulado } \\
\text { para los órganos renales. }\end{array}$ & $\begin{array}{l}\text { Células endoteliales de túbulo proximal. } \\
\text { Células endoteliales capilares peritubulares. }\end{array}$ & [30] \\
\hline & $\begin{array}{l}\text { Kidney-on-a-chip para comparar la nefrotoxicidad de la gentamicina, } \\
\text { administrada en una misma dosis, pero utilizando diferentes regíme- } \\
\text { nes farmacocinéticos. }\end{array}$ & Células epiteliales renales. & {$[31]$} \\
\hline
\end{tabular}

\section{REFERENCIAS}

[1] J. E. Hall, Guyton y Hall. Tratado de fisiología médica, 13.a ed., EUA: Elsevier, 2016.

[2] J. A. Tamayo y H. S. Lastiri, Eds., La enfermedad renal crónica en México. Hacia una política nacional para enfrentarla, México: ANMM, pp. 82, 2016.

[3] A. Milik y E. Hrynkiewicz, "On translation of LD, IL and SFC given according to IEC-61131 for hardware synthesis of reconfigurable logic controller", IFAC Proc. Vol., vol. 19, no. 1, pp. 4477-4483, 2014. DOI: 10.3182/20140824-6-za-1003.01333.
[4] IMSS, Tratamiento sustitutivo de la función renal. Diálisis y hemodiálisis en la insuficiencia renal crónica. Segundo y tercer nivel de atención (Catálogo Maestro de Guías de Práctica Clínica: IMSS-727-14). México: CENETEC, 2014. [En línea]. Disponible en: http://www. cenetec.salud.gob.mx/descargas/gpc/CatalogoMaestro/ IMSS-727-14-DialisisyhemodialisisIRC/727GER.pdf. [Consultado: 7 de agosto de 2020].

[5] CENATRA, "Reporte anual 2019 de donación y trasplantes en México", Centro Nacional de Trasplantes, México, en. 2020. [En línea]. Disponible en: https://www.gob.mx/ $\mathrm{cms} /$ uploads/attachment/file/528301/Presentacion_ anual_2019.pdf. [Consultado: 7 de agosto de 2020]. 
M. B. García-San Luis y C. Chapa-González. Avances de la Tecnología Organ-on-achip de Riñón hacia Dispositivos de Remplazo de la Función Renal

ARTÍCULO DE REVISIÓN

[6] K. J. Jang y K. Y. Suh, "A multi-layer microfluidic device for efficient culture and analysis of renal tubular cells", Lab Chip, vol. 10, no. 1, pp. 36-42, 2010. DOI: 10.1039/ b907515a.

[7] N. Ferrell, R. R. Desai, A. J. Fleischman, S. Roy, H. D. Humes y W. H. Fissell, "A microfluidic bioreactor with integrated transepithelial electrical resistance (TEER) measurement electrodes for evaluation of renal epithelial cells", Biotechnol. Bioeng., vol. 107, no. 4, pp. 707-716. 2010. DOI: 10.1002/bit.22835.

[8] A. G. Sciancalepore, F. Sallustio, S. Girardo, L. G. Passione, A. Camposeo, E. Mele, M.Di Lorenzo, V. Costantino, F. P. Schena y D. Pisignano, "A bioartificial renal tubule device embedding human renal stem/progenitor cells", PLoS One, vol. 9, no. 1, 2014. DOI: 10.1371/journal.pone.0087496.

[9] J. Sateesh, K. Guha, A. Dutta, P. Sengupta y K. Srinivasa Rao, "Design and analysis of microfluidic kidney-onchip model: fluid shear stress based study with temperature effect", Microsyst. Technol., vol. 25, no. 7, pp. 25532560, 2019. DOI: 10.1007/s00542-018-4261-z.

[10] K. Guha, J. Sateesh, A. Dutta, P. Sengupta, K. Srinivasa Rao y A. Agarwal, "Mimicking kidney re-absorption using microfluidics by considering hydrostatic pressure inside kidney tubules: structural and analytical study", Microsyst. Technol., vol. 26, no. 6, pp. 1769-1776, 2020. DOI: 10.1007/s00542-019-04720-9.

[11] H. C. Huang, Y. J. Chang, W. C. Chen, H. I. C. Harn, M. J. Tang y C. C. Wu, "Enhancement of renal epithelial cell functions through microfluidic-based coculture with adipose-derived stem cells", Tissue Eng. Part A, vol. 19, no. 17-18, pp. 2024-2034, 2013. DOI: 10.1089/ten. tea.2012.0605.

[12] P. Brakeman, S. Miao, J. Cheng, C. Z. Lee, S. Roy, W. H. Fissell y N. Ferrell, "A modular microfluidic bioreactor with improved throughput for evaluation of polarized renal epithelial cells", Biomicrofluidics, vol. 10, no. 6, pp. 1-9, 2016. DOI: 10.1063/1.4966986.

[13] C. Shen, Q. Meng y G. Zhang, "Increased curvature of hollow fiber membranes could up-regulate differential functions of renal tubular cell layers", Biotechnol. Bioeng., vol. 110, no. 8, 2013. DOI: 10.1002/bit.24874.
[14] J. Jansen, M. Fedecostante, M. J. Wilmer, J. G. Peters, U. M. Kreuser, P. H. van den Broek, R. A. Mensink, T. J. Boltje, D. Stamatialis, J. F. Wetzels, L. P. van den Heuvel, J. G. Hoenderop y R. Masereeuw, "Bioengineered kidney tubules efficiently excrete uremic toxins", Sci. Rep., vol. 6, pp. 1-12, 2016. DOI: 10.1038/srep26715.

[15] J. A. W. Jong, Y. Guo, D. Hazenbrink, S. Douka, D. Verdijk, J. van der Zwan, K. Houben, M. Baldus, K. C. Scheiner, R. Dalebout, M. C. Verhaar, R. Smakman, W. E. Hennink, K. G. F. Gerritsen y C. F. van Nostrum, "A Ninhydrin-Type Urea Sorbent for the Development of a Wearable Artificial Kidney", Macromol. Biosci., vol. 20, no. 3, 2020. DOI: 10.1002/mabi.201900396.

[16] C. P. Ng, Y. Zhuang, A. W. H. Lin y J. C. M. Teo, "A Fibrin-Based Tissue-Engineered Renal Proximal Tubule for Bioartificial Kidney Devices: Development, Characterization and In Vitro Transport Study", Int. J. Tissue Eng., vol. 2013, no. 319476, nov. 2012, pp. 1-10, 2013. DOI: $10.1155 / 2013 / 319476$.

[17] J. Vriend, J. G. P. Peters, T. T. G. Nieskens, R. Škovroňová, N. Blaimschein, M. Schmidts, R. Roepman, T. J. J. Schirris, F. G. M. Russel, R. Masereeuw y M. J. Wilmera, "Flow stimulates drug transport in a human kidney proximal tubule-on-a-chip independent of primary cilia”, Biochim. Biophys. Acta - Gen. Subj., vol. 1864, no. 1, 2020. DOI: 10.1016/j.bbagen.2019.129433.

[18] C. Sakolish, Z. Chen, C. Dalaijamts, K. Mitra, Y. Liu, T. Fulton, T. L. Wade, E. J. Kelly, I. Rusyn y W. A. Chiua, "Predicting tubular reabsorption with a human kidney proximal tubule tissue-on-a-chip and physiologically-based modeling", Toxicol. Vitr., vol. 63, no. 104752, mar. 2020. DOI: 10.1016/j.tiv.2019.104752.

[19] N. Y. C. Lin, K. A. Homan, S. S. Robinson, D. B. Kolesky, N. Duarte, A. Moisan y J. A. Lewis, "Renal reabsorption in 3D vascularized proximal tubule models", Proc. Natl. Acad. Sci. U. S. A., vol. 116, no. 12, pp. 5399-5404, 2019. DOI: $10.1073 /$ pnas.1815208116.

[20] K. A. Homan, D. B. Kolesky, M. A. Skylar-Scott, J. Herrmann, H. Obuobi, A. Moisan y J. A. Lewis, "Bioprinting of 3D Convoluted Renal Proximal Tubules on Perfusable Chips", Sci. Rep., vol. 6, pp. 1-14, 2016. DOI: 10.1038/srep34845. 
M. B. García-San Luis y C. Chapa-González. Avances de la Tecnología Organ-on-achip de Riñón hacia Dispositivos de Remplazo de la Función Renal

ARTÍCULO DE REVISIÓN

[21] J. Sateesh, K. Guha, A. Dutta, P. Sengupta, A. Agarwal y K. Srinivasa Rao, "Recreating the size-dependent reabsorption function of proximal convoluted tubule towards artificial kidney applications: Structural analysis and computational study", Artif. Organs, 2020. DOI: 10.1111/aor.13683.

[22] A. Ould-Dris, P. Paullier, L. Griscom, C. Legallais y E. Leclerc, "Analysis of the mass transfers in an artificial kidney microchip", J. Memb. Sci., vol. 352, no. 1-2, pp. 116-125, abr. 2010. DOI: 10.1016/j.memsci.2010.02.007.

[23] A. Petrosyan, P. Cravedi, V. Villani, A. Angeletti, J. Manrique, A. Renieri, R. E. De Filippo, L. Perin y S. Da Sacco, "A glomerulus-on-a-chip to recapitulate the human glomerular filtration barrier", Nat. Commun., vol. 10, no. 1, 2019. DOI: 10.1038/s41467-019-11577-z.

[24] M. Zhou, X. Zhang, X. Wen, T. Wu, W. Wang, M. Yang, J. Wang, M. Fang, B. Lin y H. Lin, "Development of a Functional Glomerulus at the Organ Level on a Chip to Mimic Hypertensive Nephropathy", Sci. Rep., vol. 6, no. 457, pp. 1-14, 2016. DOI: 10.1038/srep31771.

[25] Z. Li, L. Jiang, T. Tao, W. Su, Y. Guo, H. Yu y J. Qin, "Assessment of cadmium-induced nephrotoxicity using a kidney-on-a-chip device", Toxicol. Res. (Camb)., vol. 6, no. 3, pp. 372-380, 2017. DOI: 10.1039/c6tx00417b.

[26] S. C. Slater, V. Beachley, T. Hayes, D. Zhang, G. I. Welsh, M. A. Saleem, P. W. Mathieson, X. Wen, B. Su y S. C. Satchell, "An in vitro model of the glomerular capillary wall using electrospun collagen nanofibres in a bioartificial composite basement membrane", PLoS One, vol. 6, no. 6, 2011. DOI: 10.1371/journal.pone.0020802.

[27] S. Musah, N. Dimitrakakis, D. M. Camacho, G. M. Church y D. E. Ingber, "Directed differentiation of human induced pluripotent stem cells into mature kidney podocytes and establishment of a Glomerulus Chip", Nat. Protoc., vol. 13, no. 7, pp. 1662-1685, 2018. DOI: 10.1038/s41596-018-0007-8.

[28] C. Kensinger, S. Karp, R. Kant, B. W. Chui, K. Goldman, T. Yeager, E. R. Gould, A. Buck, D. C. Laneve, J. J. Groszek, S. Roy y W. H. Fissell, "First implantation of silicon nanopore membrane hemofilters", ASAIO J., vol. 62, no. 4, pp. 491-495, 2016. DOI: 10.1097/ MAT.0000000000000367.

[29] X. Mu, W. Zheng, L. Xiao, W. Zhang y X. Jiang, "Engineering a 3D vascular network in hydrogel for mimicking a nephron", Lab Chip, vol. 13, no. 8, pp. 1612-1618, 2013. DOI: $10.1039 / \mathrm{c} 31 \mathrm{c} 41342 \mathrm{j}$.

[30] L. Yin, G. Du, B. Zhang, H. Zhang, R. Yin, W. Zhang y S. M. Yang, "Efficient Drug Screening and Nephrotoxicity Assessment on Co-culture Microfluidic Kidney Chip", Sci. Rep., vol. 10, no. 1, pp. 1-12, 2020. DOI: 10.1038/ s41598-020-63096-3.

[31] S. Kim, S. C. LesherPerez, B. C. Kim, C. Yamanishi, J. M. Labuz, B. Leung y S. Takayama, "Pharmacokinetic profile that reduces nephrotoxicity of gentamicin in a perfused kidney-on-a-chip", Biofabrication, vol. 8, no. 1, 2016. DOI: 10.1088/1758-5090/8/1/015021.

[32] H. D. Humes, W. F. Weitzel, R. H. Bartlett, E. P. Paganini, J, R. Luderer, J. Sobota, "Initial clinical results of the bioartificial kidney containing human cells in ICU patients with acute renal failure", Kidney Int., vol. 66, no. 4, pp. 1578-1588, 2004. DOI: 10.1111/j.15231755.2004.00923.x.

[33] J. Tumlin, R. Wali, W. Williams, P. Murray, A. J. Tolwani, A. K. Vinnikova, H. M. Szerlip, J. Ye, E. P. Paganini, L. Dworkin, K. W. Finkel, M. A. Kraus y H. D. Humes, "Efficacy and safety of renal tubule cell therapy for acute renal failure", J. Am. Soc. Nephrol., vol. 19, no. 5, pp. 1034-1040, 2008. DOI: 10.1681/ASN.2007080895.

[34] V. Gura, M. B. Rivara, S. Bieber, R. Munshi, Nancy Colobong-Smith, L. Linke, J. Kundzins, M. Beizai, C. Ezon, L. Kessler y J. Himmelfarb, "A wearable artificial kidney for patients with end-stage renal disease", JCI Insight, vol. 1, no. 8, pp. 1-15, 2016. DOI: 10.1172/jci.insight.86397.

[35] M. Salani, S. Roy y W. H. Fissell, "Innovations in Wearable and Implantable Artificial Kidneys", Am. J. Kidney Dis., vol. 72, no. 5, pp. 745-751, 2018. DOI: 10.1053/j. ajkd.2018.06.005. 